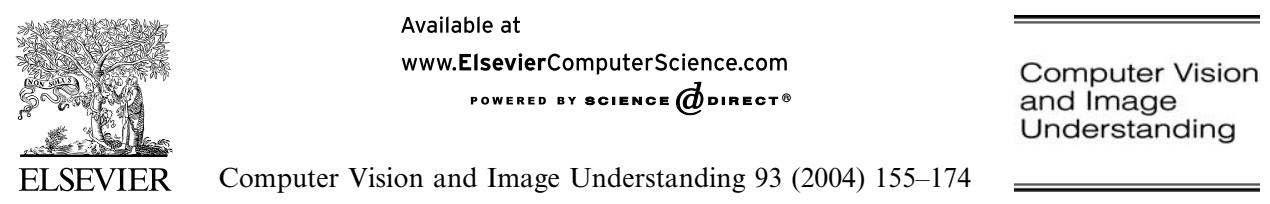

www.elsevier.com/locate/cviu

\title{
Hierarchical Markovian segmentation of multispectral images for the reconstruction of water depth maps
}

\author{
J.-N. Provost, ${ }^{\mathrm{a}, 1}$ C. Collet, ${ }^{\mathrm{a}, *}$ P. Rostaing, ${ }^{\mathrm{a}}$ P. Pérez, ${ }^{\mathrm{b}}$ \\ and P. Bouthemy ${ }^{\mathrm{b}}$ \\ a IRENav/GTS-BP 600-29240, Brest Naval, France \\ b IRISAIINRIA Rennes, Campus Universitaire de Beaulieu, 35042 Rennes Cedex, France
}

Received 15 January 2002; accepted 28 July 2003

\begin{abstract}
This paper presents an unsupervised method to segment multispectral images, involving a correlated non-Gaussian noise. The efficiency of the Markovian quadtree-based method we propose will be illustrated on a satellite image segmentation task with multispectral observations, in order to update nautical charts. The proposed method relies on a hierarchical Markovian modeling and includes the estimation of all involved parameters. The parameters of the prior model are automatically calibrated while the estimation of the noise parameters is solved by identifying generalized distribution mixtures [P. Rostaing, J.-N. Provost, C. Collet, Proc. International Workshop EMMCVPR'99: Energy Minimisation Methods in Computer Vision and Pattern Recognition, Springer Verlag, New York, 1999, p. 141], by means of an iterative conditional estimation (ICE) procedure. Generalized Gaussian (GG) distributions are considered to model various intensity distributions of the multispectral images. They are indeed well suited to a large variety of correlated multispectral data. Our segmentation method is applied to Satellite Pour l'Observation de la Terre (SPOT) remote multispectral images. Within each segmented region, a bathymetric inversion model is then estimated to recover the water depth map. Experiments on different real images have demonstrated the efficiency of the whole process and the accuracy of the obtained results has been assessed using ground truth data.

\footnotetext{
${ }^{*}$ Corresponding author. Present address: Universite Louis Pasteur, ENSPS-LSIIT UMR CNRS 7005, Bd S. Brant, 67400 Illkirch, France. Fax: +33-3-90-24-43-42.

E-mail addresses: Jean-Noel.Provost@insa-rennes.fr (J.-N. Provost), christophe.collet@ensps. u-strasbg.fr (C. Collet), bouthemy@irisa.fr (P. Bouthemy).

${ }^{1}$ Present address: IETR Groupe Image, UMR CNRS 6164, INSA-Rennes, CS 14315, 35043 Rennes Cedex, France.
} 
The designed segmentation method can be extended to images for which it is required to segment a region of interest using an unsupervised approach.

(C) 2003 Elsevier Inc. All rights reserved.

Keywords: Unsupervised segmentation; Markovian quadtree; Generalized Gaussian model; SPOT; Multispectral data; Bathymetry

\section{Introduction}

This paper is concerned with the unsupervised segmentation of satellite images. We have developed a complete process from satellite images to nautical charts. The image segmentation algorithm we propose, is based on a Hierarchical Markov Modeling [2]. Our motivations for using such a model are to provide fast computations and efficient structures to process multispectral and multiresolution large images. Indeed, computer vision community needs tools to interpret large data. Furthermore, satellites provide more and more multispectral/multiresolution images every year, so we have chosen an unsupervised approach to process efficiently a large amount of data. We focus our attention on the robustness of the algorithm.

Bathymetric surveying of shallow water environments by traditional ship-board sounding techniques is accurate but slow and expensive, particularly in geographic areas of interest that are difficult to access. Remote sensing, as already demonstrated in a wide range of earth resource issues, appears as an appealing tool for the problem under consideration. In this paper, we exploit images from the visible channels supplied by SPOT satellite to reconstruct bathymetric maps around islands or atoll lagoons (in the Indian ocean or within the atolls of the French Polynesia in the Pacific ocean). The generation and the updating of such nautical charts are of key interest for navigation or for detecting changes of coastal environment.

We have developed an original scheme consisting of three main steps. First, a segmentation step, exploiting all the channels of the SPOT image, allows us to separate the "sea area" from the others (clouds, ground). This first segmentation step will not be described in the paper: the reader is referred to [3] for details. Second, considering only segmented "sea areas," we extract the regions corresponding to similar average water depths using only two channels of the image (cf. Section 2.2). In this second segmentation step, noise modeling is handled in a specific way. We consider the class of generalized Gaussian laws which enables to accommodate a variety of observation processes within the same framework. The segmentation method is based on a Markov model in scale on a quadtree. This approach is efficient, robust and can be generalized to multispectral data [4]. Particularly, it allows us to explicitly take into account correlation between channels in the case of non-Gaussian marginal distributions [1,5]. Third, for each segmented region in the "sea area," the parameters of the bathymetric model are estimated using a robust technique. This segmentation scheme is also well suited for unsupervised segmentation of images (monospectral or multispectral), where the requirement is to focus on a region of interest. 
This paper is organized as follows. Section 2 presents the segmentation framework based on a Markov model defined on a quadtree. Section 3 is concerned with image noise modeling relying on generalized Gaussian distributions. Section 4 deals with the reconstruction of water-depth maps and reports experimental results on real images. These results demonstrate the efficiency of the proposed unsupervised segmentation method on multispectral images.

\section{Sea segmentation with a hierarchical Markov model}

Image segmentation remains a difficult problem. Statistical approaches have proved to be fruitful to design robust and efficient segmentation methods [6-9]. In the context of multispectral images, handling correlated observed data requires a well-designed modeling framework $[1,2,4,8,10,11]$. Resorting to a Bayesian scheme based on Markov models is indeed attractive when dealing with large amount of multispectral observations. Furthermore, efficient hierarchical Markov models have been proposed in the last decade [10,12,13]. Our approach relies on Markov models defined on a quadtree structure [13].

The first segmentation step of our overall scheme is completely described in [3]. Three labels or classes are considered: "land" (island, lagoon), "sea within the lagoon and open sea" and "clouds." Using a Peano path, the label map to be determined is modeled as a hidden Markov chain which is estimated from all the channels of the SPOT image. We now consider the regions labeled as "sea areas" and we aim at further segmenting them into $K$ classes, according to the observed radiometry related to water depth. A quadtree-based approach offers the well-known advantages of standard hierarchical techniques (improved robustness, ability to deal with multiresolution data), while allowing for non-iterative inference as in the case of hidden Markov chains. Besides in our context, "land" and "cloud" areas can be straightforwardly excluded from tree structure by simply masking the leaves of the quadtree where the attached observation lies (cf. Fig. 1).

\subsection{Hierarchical Markovian modeling on a quadtree}

Most of Markov models are non-causal. As a consequence, inference must be conducted iteratively, which might turn prohibitively expensive when dealing with large data images, such as multispectral images. One way to circumvent this problem is to resort to a Markov model on a quadtree where in-scale causality permits noniterative inference.

Let $G=(S, L)$ be a graph composed of a set $S$ of nodes and a set $L$ of edges. A tree is a connected graph with no cycle, where as a consequence, each node apart from the root $r$ has a unique predecessor, its "parent," on the path to the root. A quadtree, as illustrated in Fig. 1, is a special case of tree where each node, apart from the terminal ones, the "leaves," has four "children." The set of nodes $S$ can be partitioned into "scales," $S=S^{0} \cup S^{1} \cup \cdots \cup S^{R}$, according to the path length from each node to the root. Thus, $S^{R}=\{r\}, S^{n}$ involves $4^{R-n}$ sites, and $S^{0}$ is the finest scale formed by 


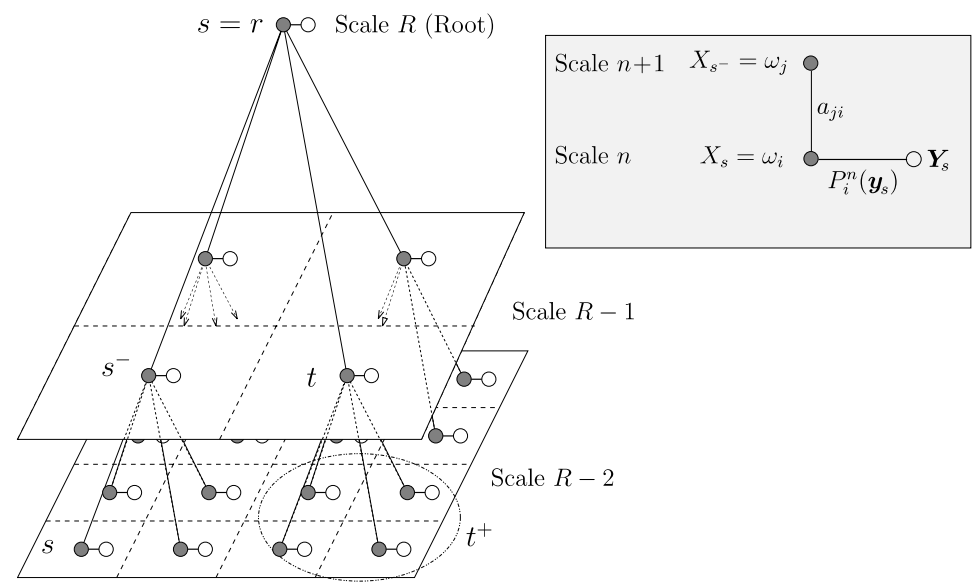

Fig. 1. Dependency graph corresponding to a quadtree structure. Black circles represent labels and white circles represent observations. The segmentation algorithm re-estimates iteratively the parameters of a given hidden in-scale-Markov model to produce a new model which has a higher probability of generating the given observation sequence. This re-estimation procedure is continued until no more significant improvement in parameters can be obtained. The two-step computation of posterior marginals propagates available information all over the tree: on one hand, the bottom-up step spreads the influence of data to other levels up to the root, on the other hand the top-down step computes the posterior marginals taking into account this information. Thus, this proposed modeling scheme captures, over the quadtree, significant statistical dependencies and provides a robust algorithm of segmentation.

the leaves. We consider a labeling process $X$ which assigns a class label $X_{s}$ to each node of $G$ :

$$
X=\left\{X^{n}\right\}_{n=0}^{R} \text { with } X^{n}=\left\{X_{s}, s \in S^{n}\right\},
$$

where $X_{s}$ takes its values in the set $\Delta=\left\{\omega_{1}, \ldots, \omega_{K}\right\}$, of the $K$ bathymetric classes. A number of conditional independence properties are assumed.

First, $X$ is supposed to be Markovian in scale, i.e., ${ }^{2}$

$$
P\left(x^{n} \mid x^{k}, k>n\right)=P\left(x^{n} \mid x^{n+1}\right) .
$$

It is also assumed that the probabilities of inter-scale transitions can be factorized in the following way $[13,14]$ :

$$
P\left(x^{n} \mid x^{n+1}\right)=\prod_{s \in S^{n}} P\left(x_{s} \mid x_{s^{-}}\right),
$$

where $s^{-}$designates the father of site $s$, as illustrated in Fig. 1 .

Finally, the likelihood of the observations $\boldsymbol{Y}$ conditionally to $X$ is expressed as the following product (assuming conditional independence):

\footnotetext{
${ }^{2}$ To simplify notation, we will denote the discrete probability $P(X=x)$ as $P(x)$.
} 


$$
P(\boldsymbol{y} \mid x)=\prod_{n=0}^{R} P\left(\boldsymbol{y}^{n} \mid x^{n}\right)=\prod_{n=0}^{R} \prod_{s \in S^{n}} P\left(\boldsymbol{y}_{s} \mid x_{s}\right),
$$

where $\forall s \in S^{n}, \forall n \in\{0, \ldots, R\}, P\left(\boldsymbol{y}_{s} \mid x_{s}=\omega_{i}\right) \triangleq P_{i}^{n}\left(\boldsymbol{y}_{s}\right)$, captures the likelihood of the data $y_{s}$. Each site $s$ of scale $n$ can be associated with a label $\omega_{i}$.

We suppose now that data are only available at the finest level $(n=0)$. When no observation exists $(n>0)$ or when the site at scale 0 does not belong to an area labeled as "sea," the likelihood $P_{i}^{n}\left(\boldsymbol{y}_{s}\right)$ is set to 1 . If, in a specific application, we have images of the same area at different levels of resolution, the quadtree structure can be still used and permits to properly consider all the available data. It is a way to conduct the segmentation while merging data.

From these assumptions, it can be easily inferred that the joint distribution $P(x, y)$ follows a Gibbs law whose expression is given by [13]

$$
P(x, y)=P\left(x_{r}\right) \prod_{s \neq r} P\left(x_{s} \mid x_{s^{-}}\right) \prod_{s \in S} P\left(\boldsymbol{y}_{s} \mid x_{s}\right) .
$$

One of the interests of this model lies in the possibility of computing exactly the posterior marginals $P\left(X_{s} \mid \boldsymbol{Y}\right)$ and $P\left(X_{s}, X_{s}^{-} \mid \boldsymbol{Y}\right)$ at each node $s$ within two passes. These computed expressions will be first used in the iterative parameter estimation step, as described in the next section. The segmentation label map $\hat{x}$ to be determined is finally given by:

$$
\hat{\boldsymbol{x}}_{s}=\arg \max _{\omega_{i} \in \Delta} P\left(X_{s}=\omega_{i} \mid \boldsymbol{Y}=\boldsymbol{y}\right) .
$$

Eq. (6) shows that we obtain a labeling of each pixel at each level of the quadtree, even if observations only lie on the finest level. Indeed, the two-step computation of posterior marginals (described in Table 1) propagates available information all over the tree. The bottom-up step spreads the influence of data to other levels up to the root. Then, the top-down step computes the posterior marginals taking into account this information. Of course, if observations are available for other nodes of the tree, this algorithm merges all the observations to obtain a more accurate segmentation result. The root of the quadtree is also a node of the pyramid. Therefore we have a segmentation result for this node too. This result is obtain (like for each node) by maximizing posterior marginals (cf. Eq. (6)). Experimental results show that $\hat{x}_{r}$ represents the most frequent class of the image.

\subsection{Application to water depth maps}

As mentioned in the introduction, to illustrate the efficiency of our algorithm, we apply it to SPOT images. Those satellite images are acquired in three spectral bands: $\mathrm{XS}_{1}$ channel covering $0.50-0.59 \mu \mathrm{m}$ (green), $\mathrm{XS}_{2}$ channel covering $0.61-0.68 \mu \mathrm{m}$ (red), and $\mathrm{XS}_{3}$ channel covering $0.79-0.89 \mu \mathrm{m}$ (near infrared). It is generally assumed that the solar radiation is exponentially attenuated with depth by the water column and thus the observed radiances follow the same law $[15,16]$. Channels $\mathrm{XS}_{1}-\mathrm{XS}_{2}$ are usable for coastal mapping as they penetrate water up to 25-30 meters and 7-9 meters, 
Table 1

Two-pass computation of posterior marginals on the quadtree (with notations from Fig. 1)

1. Evaluation of the partial posterior marginals ${ }^{\mathrm{a}}$ at full resolution $(n=0)$ :

$$
P\left(X_{s}=\omega_{i} \mid \boldsymbol{y}_{\geqslant s}\right)=P\left(X_{s}=\omega_{i} \mid \boldsymbol{y}_{s}\right)=\frac{P\left(X_{s}=\omega_{i}\right) P_{i}^{0}\left(\boldsymbol{y}_{s}\right)}{\sum_{j} P\left(X_{s}=\omega_{j}\right) P_{j}^{0}\left(\boldsymbol{y}_{s}\right)} \quad \forall s \in S^{0},
$$

where $P\left(X_{s}=\omega_{i}\right)$ is recursively evaluated through a top-down pass according to for $n=R-1 \ldots 0$

$$
\mid P\left(X_{s}=\omega_{i}\right)=\sum_{j} a_{j i} P\left(X_{s^{-}}=\omega_{j}\right) \quad \forall s \in S^{n}
$$

Note that the a priori probability $P\left(X_{r}=\omega_{i}\right)=\pi_{i}$ initializes the procedure (cf. Fig. 1).

2. Bottom-up recursion on partial posterior marginals ${ }^{\mathrm{b}}$

for $n=1 \ldots R$

$$
\mid \begin{aligned}
& P\left(X_{s}=\omega_{i} \mid \boldsymbol{y}_{\geqslant s}\right)=\frac{1}{Z} P\left(X_{s}=\omega_{i}\right)^{-3} P_{i}^{n}\left(\boldsymbol{y}_{s}\right) \prod_{t \in s^{+}} \sum_{j} a_{i j} \frac{P\left(X_{s}=\omega_{i}\right)}{P\left(X_{t}=\omega_{j}\right)} P\left(X_{t}=\omega_{j} \mid \boldsymbol{y}_{\geqslant t}\right) \\
& \forall s \in S^{n}
\end{aligned}
$$

where $Z$ is a normalizing factor satisfying the condition $\sum_{i} P\left(X_{s}=\omega_{i} \mid \boldsymbol{y}_{\geqslant s}\right)=1$. At the end of the recursion, we obtain the a posteriori probability for the $\operatorname{root} \xi_{r}(i)=P\left(X_{r}=\omega_{i} \mid \boldsymbol{y}\right)$, which initializes the next step.

3. Top-down recursion on posterior marginals ${ }^{c}$ for $n=R-1 \ldots 0$

$$
\mid \begin{aligned}
& \Psi_{s}(i, j)=P\left(X_{s}=\omega_{j}, X_{s^{-}}=\omega_{i} / \boldsymbol{y}\right)=\xi_{s^{-}}(i) \frac{P\left(X_{s}=\omega_{i} \mid \boldsymbol{y}_{\geqslant s}\right) a_{i j} P\left(X_{s^{-}}=\omega_{i}\right) / P\left(X_{s}=\omega_{j}\right)}{\sum_{l} P\left(X_{s}=\omega_{l} \mid \boldsymbol{y}_{\geqslant s}\right) a_{i l} P\left(X_{s^{-}}=\omega_{i}\right) / P\left(X_{s}=\omega_{l}\right)} \quad \forall s \in S^{n} \\
& \xi_{s}(j)=P\left(X_{s}=\omega_{j} \mid \boldsymbol{y}\right)=\sum_{i} \Psi_{s}(i, j)
\end{aligned}
$$

${ }^{\mathrm{a}}$ The symbol $\geqslant s$ denotes all the descendents of $s, s$ included.

${ }^{\mathrm{b}}$ The symbol $s^{+}$stands for the 4 children (level $n-1$ ) of the site $s$ (level $n$ ).

${ }^{\mathrm{c}}$ The symbol $s^{-}$stands for the single parent (level $\left.n+1\right)$ of the site $s($ level $n$ ).

respectively. Channel $\mathrm{XS}_{3}$ cannot be used since the associated wavelength does not penetrate into the water beyond few centimeters. Hence, we consider images from channels $\mathrm{XS}_{1}$ and $\mathrm{XS}_{2}$ whose radiance values are not only dependent on water depth but may be influenced by the nature of the sea-bottom or the water turbidity as well. Afterwards, each "sea" observation $y_{s}$ at site $s$ of scale $n=0$ is formed by two values from XS1 and XS2 channels. One contribution of our work is to apply the segmentation algorithm only to some pixels of the original image. This method can be applied in many other applications to segment a part of an image extracted by a pre-treatment.

\subsection{Parameter estimation step}

Segmenting an image into different regions or classes and estimating all the model parameters are intricate problems. Fixing or estimating correctly model parameters is crucial in practice to get an accurate and reliable segmentation. Here, we are dealing with an unsupervised labelling problem where parameters $\Phi=\left\{\Phi_{x}, \Phi_{y}\right\}$ must be estimated: 
- $\Phi_{y}$ stands for the parameters involved in the data model. The conditional data likelihood (i.e., data driven term) is entirely defined by $P_{i}^{0}\left(\boldsymbol{y}_{s}\right)=P\left(\boldsymbol{Y}_{s}=\right.$ $\left.\boldsymbol{y}_{s} \mid x_{s}=\omega_{i}, s \in S^{0}\right)$ since the only observations available (corresponding to XS1 and XS2 channels) are associated to the leaves of the label tree. In this context, several models of image noise could be used [1,5]. We have considered the Generalized Gaussian (GG) model as described in Section 3, in order to cope with a large variety of situations;

- $\Phi_{x}=\left(\left(a_{i j}\right)_{i, j=1}^{K},\left(\pi_{i}\right)_{i=1}^{K}\right)$ is the set of the prior Markov model parameters where $a_{i j} \triangleq P\left(X_{s}=\omega_{j} \mid X_{s^{-}}=\omega_{i}\right)$ and $\pi_{i} \triangleq P\left(X_{r}=\omega_{i}\right)$.

We estimate these two sets of parameters using an iterative conditional estimation (ICE) method [17]. This method requires to find two estimators, namely $\widehat{\Phi}_{x}=\Phi_{x}(X)$ and $\widehat{\Phi}_{y}=\Phi_{y}(X, \boldsymbol{Y})$. When $X$ is unobservable (i.e., incomplete data), the iterative ICE procedure introduces the estimates $\widehat{\Phi}_{x}^{[k+1]}$ and $\widehat{\Phi}_{y}^{[k+1]}$ at step $k+1$ defined as the conditional expectations of $\Phi_{x}$ and $\Phi_{y}$ given $\boldsymbol{Y}=\boldsymbol{y}$, and the current parameter values $\widehat{\Phi}_{x}^{[k]}$ and $\widehat{\Phi}_{y}^{[k]}$ :

$$
\widehat{\Phi}^{[k+1]}=E\left[\widehat{\Phi} / \boldsymbol{Y}=\boldsymbol{y}, \widehat{\Phi}^{[k]}\right] .
$$

They are the best approximations of $\Phi_{x}$ and $\Phi_{y}$ w.r.t. the mean square error. When $E[\widehat{\Phi} / \boldsymbol{Y}=\boldsymbol{y}]$ is not calculable, but sampling from the posterior distribution $P(X \mid \boldsymbol{Y}, \Phi)$ is achievable, one can utilize a stochastic approximation. In our case, the tree structure enables the exact computation of the posterior marginals. It is thus possible to determine the exact update of the prior parameter values [13], where $\widehat{\Phi}_{x}$ is the maximum likelihood (ML) estimator:

$$
\left\{\begin{array} { l } 
{ a _ { i j } = \frac { \sum _ { s \neq r } \Psi _ { s } ( i , j ) } { \sum _ { s \neq r } \xi _ { s ^ { - } } ( i ) } } \\
{ \pi _ { i } = \xi _ { r } ( i ) }
\end{array} \quad \text { with } \left\{\begin{array}{l}
\xi_{s}(i) \triangleq P\left(X_{s}=\omega_{i} / \boldsymbol{Y}=\boldsymbol{y}, \Phi^{[k]}\right), \\
\Psi_{s}(i, j) \triangleq P\left(X_{s}=\omega_{j}, X_{s^{-}}=\omega_{i} / \boldsymbol{Y}=\boldsymbol{y}, \Phi^{[k]}\right) .
\end{array}\right.\right.
$$

The complete procedure including the two-pass computation of posterior marginals $\Psi_{s}$ and $\xi_{s}$ is presented in Table 1 . The ICE algorithm realizes a deterministic estimation of the a priori parameters (Eq. (8)), whereas the data-driven parameters $\Phi_{y}$ are updated in a stochastic way [7,13] using samples from $P\left(X \mid \boldsymbol{Y}=\boldsymbol{y}, \Phi^{[k]}\right)$. The noise model is now described in the following section.

\section{Noise modeling}

As image noise model, we consider the general class of Generalized Gaussian (GG) distributions $\mathcal{G}$ [18] whose expression is given by:

$$
\mathcal{G}\left(z / \mu, \sigma^{2}, p\right)=[2 \Gamma(1 / p)]^{-1} \eta(p) p \exp \left[-(\eta(p)|z-\mu|)^{p}\right],
$$

where $\Gamma($.$) is the Gamma function, p$ is a positive shape parameter governing the rate of decay ( $p=1$ for Laplacian noise, $p=2$ for Gaussian noise, $p>8$ for nearly uniform noise), $\mu$ is the mean, and 


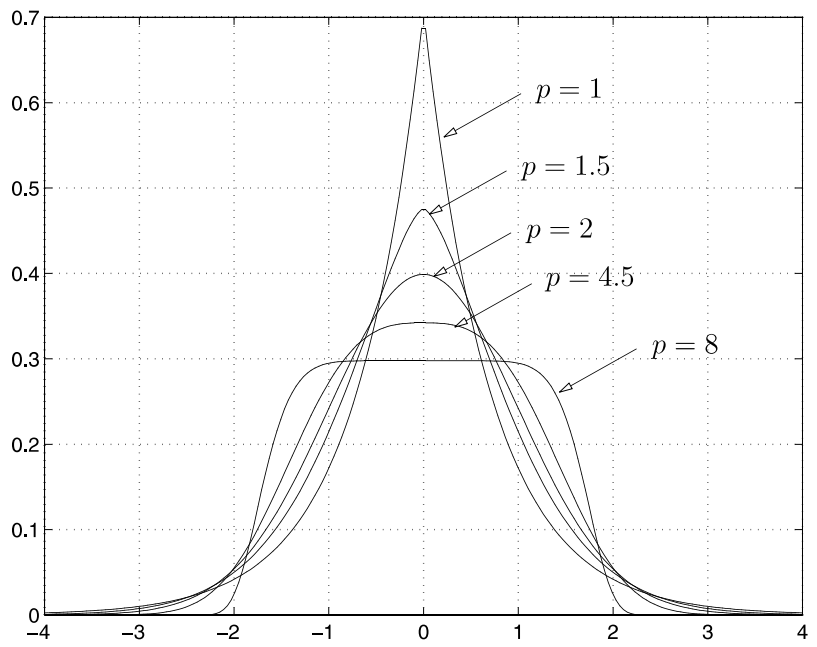

Fig. 2. Plot of generalized Gaussian probability density functions for several values of the shape parameter $p$ with $\sigma^{2}=1, \mu=0$.

$$
\eta(p) \triangleq\left[\frac{\Gamma(3 / p)}{\sigma^{2} \Gamma(1 / p)}\right]^{1 / 2},
$$

where $\sigma^{2}$ is the variance. Let us note that for small values of $p$ (i.e., $p<2$ ), this probability density function has heavier tail than Gaussian density (cf. Fig. 2). This noise model is well adapted to model a large range of physical processes, and has been recently used in different contexts such as underwater acoustic noise modeling [19], multiresolution image denoising schemes [20], or watermarking application [21].

\subsection{Estimation of the parameters of the $G G$ pdf on complete data}

The properties of the maximum likelihood (ML) estimation of the generalized Gaussian pdf parameters from $M$ independent identically distributed samples $\left\{z_{1}, \ldots, z_{M}\right\}$, are investigated in [22]. The estimation of parameters $p$ and $\sigma$ (assuming that $\mu$ is known) is straightforward by solving simultaneously the likelihood equations: $\left(\partial \mathcal{G}\left(z / \mu, \sigma^{2}, p\right)\right) /\left(\partial \sigma^{2}\right)=0$ and $\left(\partial \mathcal{G}\left(z / \mu, \sigma^{2}, p\right)\right) /(\partial p)=0$. For parameter $p$, the resulting equation to be numerically solved is:

$$
p+\Psi(1 / p)+\log (p / M)+\log G_{p}(\tilde{z})-p \frac{G_{p}^{\prime}(\tilde{z})}{G_{p}(\tilde{z})}=0,
$$

where $\Psi($.$) is the di-gamma function [23], G_{p}(\tilde{z})$ is the $p$-norm ${ }^{3}$ of the vector $\tilde{\boldsymbol{z}}=\boldsymbol{z}-\mu I$ (with $\boldsymbol{z}=\left[z_{1}, \ldots, z_{M}\right]^{\mathrm{T}}$ and $I=\left[\begin{array}{lll}1 & 1 \cdots 1\end{array}\right]^{\mathrm{T}}$ ) to the power of $p$ (i.e., $\left.G_{p}(\tilde{z})=\left(\|\tilde{\boldsymbol{z}}\|_{p}\right)^{p}\right)$, and $G_{p}^{\prime}(\tilde{z})$ is the derivative of $G_{p}(\tilde{z})$ with respect to $p$.

\footnotetext{
${ }^{3}$ The $p$-norm of the vector $\tilde{\boldsymbol{z}}=\left[\tilde{z}_{1}, \ldots, \tilde{z}_{M}\right]^{\mathrm{T}}$ is defined by $\|\tilde{\boldsymbol{z}}\|_{p}=\left(\sum_{k=1}^{M}\left|\tilde{z}_{k}\right|^{p}\right)^{1 / p}$.
} 
The solution $\widehat{p}$ is then substituted for in the equation $\left(\partial \mathcal{G}\left(z / \mu, \sigma^{2}, p\right)\right) /\left(\partial \sigma^{2}\right)=0$ to yield the estimation $\widehat{\sigma}^{2}$ of parameter $\sigma^{2}$ :

$$
\widehat{\sigma}^{2}=\frac{\Gamma(3 / \widehat{p})}{\Gamma(1 / \widehat{p})}\left[\left(\frac{\hat{p}}{M}\right)^{1 / \hat{p}}\|\tilde{z}\|_{\hat{p}}\right]^{2} .
$$

The estimation of the mean $\mu$ is more complex. The Maximum Likelihood estimator of $\mu$ (assuming $p$ and $\sigma$ known) can be written as [22]:

$$
\widehat{\mu}=J_{p}^{\mathrm{T}} \boldsymbol{z}
$$

where $J_{p}$ is the generalized $l_{p}$ inverse of $I$, i.e., the vector for which the $p$-norm $\left\|I J_{p}^{\mathrm{T}} \boldsymbol{z}-\boldsymbol{z}\right\|_{p}$ is minimized. For the special case where $p=2, J_{p}$ corresponds to the pseudo-inverse of $I$ (i.e., $J_{p}=I / M$ ).

By solving simultaneously (11) and (13), we obtain the ML estimators of $p$ and $\mu$. Since this procedure is computationally expensive, we prefer to evaluate the parameter $\mu$ by using the empirical mean given by:

$$
\widehat{\mu}=\frac{1}{M} \sum_{k=1}^{M} z_{k} .
$$

Let us note that the empirical estimator of the parameter $\mu$ of the generalized Gaussian pdf is unbiased and consistent [24].

To conclude, let us summarize the successive steps to estimate the GG pdf parameters. First, the mean $\mu$ is estimated according to relation (14), then the "shape" parameter $p$ is numerically estimated from Eq. (11), and finally the variance is estimated using expression (12).

\subsection{Image noise model used for the multispectral data}

Conditionally to $X_{s}=\omega_{i}$, the covariance matrix of the observation vector ${ }^{4} \boldsymbol{Y}_{s}$, is positive-definite symmetric and admits the following Crout (Cholesky) factorization:

$$
\Sigma_{i}^{y} \triangleq\left[\begin{array}{ll}
\left(\varsigma_{i}^{(1)}\right)^{2} & \rho_{i} \varsigma_{i}^{(1)} \varsigma_{i}^{(2)} \\
\rho_{i} \varsigma_{i}^{(1)} \varsigma_{i}^{(2)} & \left(\varsigma_{i}^{(2)}\right)^{2}
\end{array}\right]=L_{i} L_{i}^{\mathrm{T}},
$$

where $\left(\left(\varsigma_{i}^{(1)}\right),\left(\varsigma_{i}^{(2)}\right), \rho_{i}\right)$ stands, respectively, for the standard deviation on channels B and $\mathrm{G}$ and correlation factor, all on $i$ th class. $L_{i}$ is the lower triangular matrix

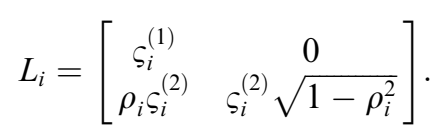

\footnotetext{
${ }^{4} \boldsymbol{Y}_{s}=\left[Y_{s}^{(1)} Y_{s}^{(2)}\right]^{\mathrm{T}}$, the superscripts (1) and (2) correspond, respectively, to SPOT channels B $\left(\mathrm{XS}_{1}\right)$ and $\mathrm{G}\left(\mathrm{XS}_{2}\right)$, respectively.
} 
With this factorization, the correlated random vector $\boldsymbol{Y}_{s}$ can be transformed into an uncorrelated vector $\boldsymbol{Z}_{s}$ defined by:

$$
\boldsymbol{Z}_{s}=A_{i} \boldsymbol{Y}_{s} \quad \text { with } \quad A_{i} \triangleq L_{i}^{-1}=\left[\begin{array}{cc}
\frac{1}{\varsigma_{i}^{(1)}} & 0 \\
-\frac{\rho_{i}}{\varsigma_{i}^{(1)} \sqrt{1-\rho_{i}^{2}}} & \frac{1}{\varsigma_{i}^{(2)} \sqrt{1-\rho_{i}^{2}}}
\end{array}\right] .
$$

We assume that the two components of $\boldsymbol{Z}_{s}$ are independent conditionally to $X_{s}=\omega_{i}$ and that both follow two GG pdfs with parameters $\left(\mu_{i}^{(1)}, \sigma_{i}^{(1)}, \rho_{i}^{(1)}\right)$ and $\left(\mu_{i}^{(2)}, \sigma_{i}^{(2)}, \rho_{i}^{(2)}\right)$, respectively.

From (17), we get ${ }^{5} \boldsymbol{\mu}_{i}=A_{i} \boldsymbol{m}_{i}$ and the GG pdfs of $\boldsymbol{Z}_{s}$ and $\boldsymbol{Y}_{s}$ are linked by the relation:

$$
\begin{aligned}
\mathcal{G}\left(\boldsymbol{y}_{s}\right)= & \operatorname{det}\left(A_{i}\right) \mathcal{G}\left(A_{i} \boldsymbol{y}_{s} \mid \boldsymbol{\mu}_{i}, \boldsymbol{\sigma}_{i}, \boldsymbol{p}_{i}\right) \\
= & \frac{1}{\varsigma_{i}^{(1)} \varsigma_{i}^{(2)} \sqrt{1-\rho_{i}^{2}}} \mathcal{G}\left(\frac{y_{s}^{(1)}}{\varsigma_{i}^{(1)}} / \mu_{i}^{(1)}, \sigma_{i}^{(1)}, p_{i}^{(1)}\right) \\
& \times \mathcal{G}\left(-\frac{y_{s}^{(1)} \rho_{i} \varsigma_{i}^{(2)}-y_{s}^{(2)} \varsigma_{i}^{(1)}}{\varsigma_{i}^{(1)} \varsigma_{i}^{(2)} \sqrt{1-\rho_{i}^{2}}} / \mu_{i}^{(2)}, \sigma_{i}^{(2)}, p_{i}^{(2)}\right)
\end{aligned}
$$

This decorrelation technique of the multispectral observed data was introduced in [5] by assuming that decorrelated observed data are conditionally independent and that their pdfs belong to a restricted set of probability functions (Gaussian, Laplacian, etc). We have extended it to the case of generalized Gaussian pdfs. The GG pdfs will now model the conditional marginal likelihoods $P_{i}^{0}\left(\boldsymbol{y}_{s}\right)=P\left(\boldsymbol{Y}_{s}=\boldsymbol{y}_{s} \mid x_{s}\right.$ $\left.=\omega_{i}, s \in S^{0}\right)$.

\subsection{Evaluation of the local likelihood $P_{i}^{0}$ for discrete observations}

In practice, numerical radiometry values are supplied for each channel (e.g., 8 bits per channel). Let us consider the numerical evaluation of marginal likelihood $P_{i}^{0}\left(\boldsymbol{y}_{s}\right)$ which is required in the segmentation algorithm. It is crucial in order to really exploit the benefit of this "elaborate" noise modeling. Indeed, for small values of $p$ (i.e., impulsive noise $p<2$ ), the numerical estimation of GG pdf needs to be accurate enough. Hence, the corresponding joined probability of $\boldsymbol{Y}_{s}=(l, m)$ for $l, m \in\{0, \ldots, 255\}$ given $X_{s}=\omega_{i}$ is computed from a sampled lattice grid around $(l, m)$ of given size $(2 N+1) \times(2 J+1)$ (cf. Fig. 3) as:

$$
\begin{aligned}
P\left(\boldsymbol{y}_{s}\right. & \left.=(l, m) \mid X_{s}=\omega_{i}\right) \\
& =\frac{1}{(2 N+1)(2 J+1)} \sum_{n=-N}^{N} \sum_{j=-J i}^{J} \mathcal{G}_{i}\left(l+\frac{n}{2 N+1}, m+\frac{j}{2 J+1}\right),
\end{aligned}
$$

where $\mathcal{G}_{i}$ is the likelihood of $\boldsymbol{Y}_{s}$ conditioned on $X_{s}=\omega_{i}$ given in (18), whereas $P\left(\boldsymbol{y}_{s}=(l, m) \mid X_{s}=\omega_{i}\right)$ stands for the probability used by the segmentation algorithm.

\footnotetext{
${ }^{5} \mu_{i}=\left[\mu_{i}^{(1)} \mu_{i}^{(2)}\right]^{\mathrm{T}}$ is the mean of $\boldsymbol{Z}_{s}$ given $X_{s}=\omega_{i}$ and $\boldsymbol{m}_{i}=\left[m_{i}^{(1)} m_{i}^{(2)}\right]^{\mathrm{T}}$ is the mean of $\boldsymbol{Y}_{s}$ given $X_{s}=\omega_{i}$.
} 


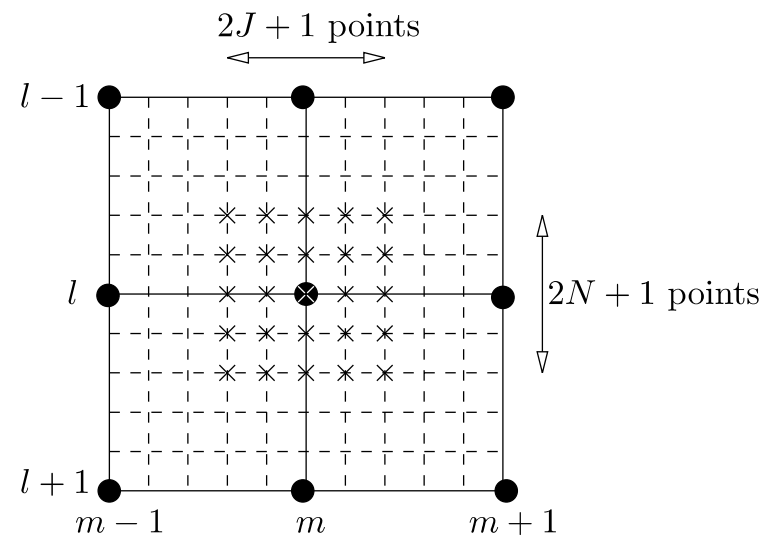

Fig. 3. Point resampling grid to estimate the probability $P\left(\boldsymbol{y}_{s}=(l, m) \mid x_{s}=\omega_{i}\right)$. This is an important ingredient when the likelihood function has a thin shape compared to the distance between two gray levels (cf. Eq. (19)).

\section{Application to the generation of nautical charts}

In order to demonstrate the efficiency of the segmentation method, we now apply it to the reconstruction of water depth maps from multispectral SPOT images. We first report results of segmentation using the statistical method described in Sections 2 and 3 involving a prior Markov model defined on a quadtree and GG pdfs to model the conditional likelihood of the image observations. Then, we will introduce the bathymetric inversion model used. From the bathymetry application point of view, a key contribution of our work lies in the independent estimation of these parameters for each water depth class resulting from the segmentation of the sea areas.

\subsection{Segmentation of SPOT images}

We summarize in Table 2 the overall segmentation method relying on a prior Markov model defined on a quadtree (described in Section 2.1) and a GG image noise model (described in Section 3.2) and exploiting two channels of one SPOT image.

Fig. 4B shows the obtained segmentation map into three classes for the original SPOT image displayed in Fig. 4A. Table 3 contains the estimated values of the "shape" parameter $p$ for each class. Let us note that, for class $\omega_{2}$, the two estimated GG pdfs are (almost) Gaussian, whereas for the two other classes, the estimated GG noise models exhibit low "shape" parameter values corresponding to heavy tail densities.

In order to validate the GG noise model, the following Kolmogorov test $\mathcal{K}_{i}$ is computed over each segmented region:

$$
\mathcal{K}_{i}=\frac{1}{N_{i}} \sum_{l, m=0}^{255}\left|H_{i}(l, m)-N_{i} P\left(\boldsymbol{y}_{s}=(l, m) \mid X_{s}=\omega_{i}\right)\right|,
$$


Table 2

Outline of the overall unsupervised algorithm from initialization to final segmentation

I Parameter initialization

1. Priors $\left(\Phi_{x}^{[0]}\right): \pi_{i}=1 / K \quad$ and $\quad a_{i j}=\left\{\begin{array}{ll}\frac{1}{2} & \text { for } i=j, \\ \frac{1}{2(K-1)} & \text { otherwise. }\end{array}\right.$ with $K$ the number of classes.

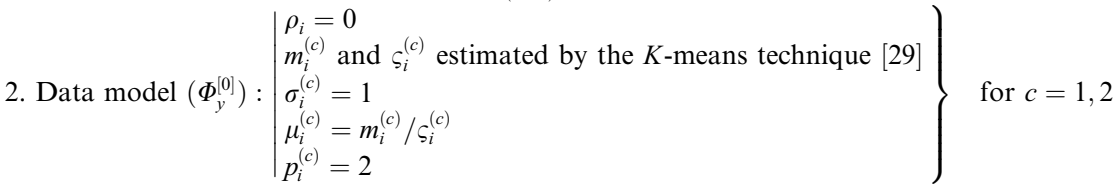

The probability $P_{i}^{n}\left(\boldsymbol{y}_{s}\right)$ is computed using Eqs. (18) and (19) for $n=0$, and $P_{i}^{n}\left(\boldsymbol{y}_{s}\right)=1$ elsewhere.

II Estimation procedure: two-pass computation of posterior marginals $P\left(X_{s}=\omega_{i} \mid \boldsymbol{y}, \Phi^{[k]}\right)$ and $P\left(X_{s}=\omega_{j}, X_{s}^{-}=\omega_{i} \mid \boldsymbol{y}, \Phi^{[k]}\right)($ Table 1$)$.

III Parameter updating:

1. The parameters $\pi_{i}^{[k]}$ and $a_{i j}^{[k]}$ are evaluated using Eq. (8).

2. Data parameters are updated using the following stochastic procedure:

(a) draw a sample from $P\left(X_{s}=\omega_{i} \mid \boldsymbol{y}, \Phi^{[k]}\right)$ for $s \in S^{0}$

(b) Covariance matrix estimation:

$$
\hat{\boldsymbol{\Sigma}}_{i}^{y}=\frac{1}{N_{i}} \sum_{\left\{s \in S^{0} / X_{s}=\omega_{i}\right\}}\left(\boldsymbol{y}_{s}-\boldsymbol{m}_{i}\right)\left(\boldsymbol{y}_{s}-\boldsymbol{m}_{i}\right)^{\mathrm{T}}, \quad \text { where } N_{i}=\operatorname{card}\left\{X_{s}=\omega_{i} \text { for } s \in S^{0}\right\} .
$$

(c) Decorrelation procedure (Eq. (17)).

(d) Estimation of the GG pdf parameters in the decorrelated space: $\mu_{i}^{(c)}$ (Eq. (14)), $p_{i}^{(c)}$ (Eq. (11)) and $\sigma_{i}^{(c)}$ (Eq. (12)) for $c=1$ and 2 .

(e) Evaluation of the likelihood $P\left(\boldsymbol{y}_{s} \mid X_{s}=\omega_{i}, \Phi_{y}^{[k+1]}\right)$ using Eqs. (18) and (19).

IV Repeat step II until $\Phi_{x}^{[k]}$ and $\Phi_{y}^{[k]}$ converge

$V$ Segmentation step:

The modes of a posteriori marginals (MPM) provide the final segmentation (Eq. (6)).

where $H_{i}(l, m)$ is the two-dimensional histogram of the observed data at pixel $s$ labeled by class $\omega_{i}$ and $N_{i}$ is the number of points belonging to class $\omega_{i}$.

In Table 4, we report the computed $\mathcal{K}_{i}$-test values for the three classes $\left\{\omega_{1}, \omega_{2}, \omega_{3}\right\}$, both for the Gaussian model and for the GG model. The segmentation of sea areas into three classes can also be obtained when considering Gaussian models by simply fixing the shape parameters to 2. These results (second column of Table 4) illustrate the better adequacy of the GG noise model compared to the Gaussian one. Let us point out that we obtain similar values for the class $\omega_{2}$. Indeed, for this class, both shape parameter values are close to 2 when considering the GG model (Table 3).

\subsection{Reconstruction of water depth information}

\subsubsection{SPOT radiometry and bathymetry}

A way to establish a relation between radiometry and bathymetry was initially proposed by Lyzenga [16,15]. It implies that the sea bottom is uniform over the area considered for the computation of the bathymetry model [25]. 


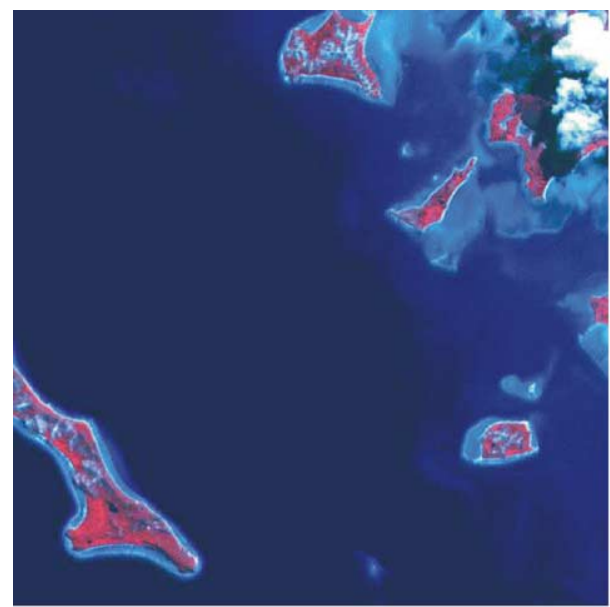

$\mathbf{A}$

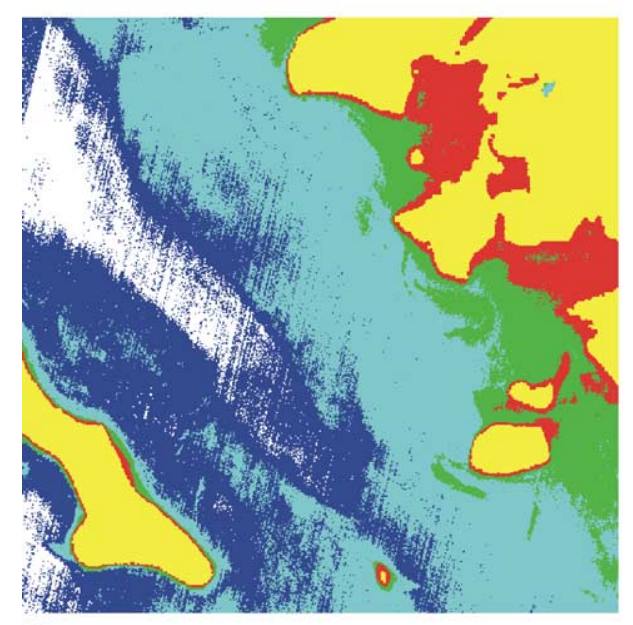

C

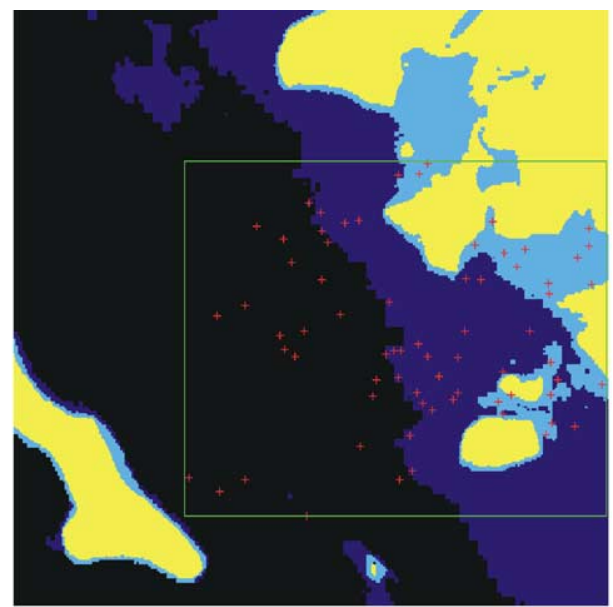

B

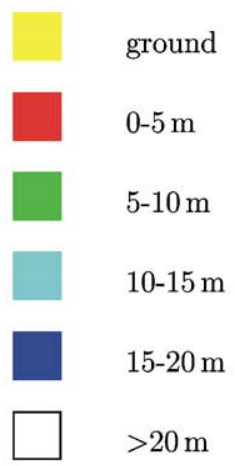

Fig. 4. (A) Acquired $512 \times 512$ RGB SPOT Image. One observes the ground areas (in red color, due to infrared radiation of the vegetation), white zones corresponding to clouds reflection in the three bands, and sea areas with different blue levels, corresponding to the penetration of $\left(\mathrm{XS}_{1}-\mathrm{XS}_{2}\right)$ bands within the water. (B) Segmentation results obtained using a quadtree structure and the generalized Gaussian noise model. Three water depth classes where defined $(K=3)$. The red crosses correspond to the set of in situ bathymetric measurement locations (class $\omega_{1}$ (black): 20 points, class $\omega_{2}$ (dark blue): 30 points, class $\omega_{3}$ (light blue): 20 points). The yellow color stands for ground zones. (C) Nautical chart generated by the class-wise bathymetric models estimated for each segmented region and using the training set of in situ bathymetric measurements.

As far as we are concerned, we estimate one bathymetric model for each class $\left\{\omega_{1}, \omega_{2}, \omega_{3}\right\}$ resulting from the segmentation stage, by using the following relation [15,25]: 
Table 3

Estimation of the "shape" parameter $p$ on the decorrelated data

\begin{tabular}{lll}
\hline Class $\omega_{i}$ & $p^{(1)}$ & $p^{(2)}$ \\
\hline$\omega_{1}$ & 1.3 & 1.7 \\
$\omega_{2}$ & 2.0 & 2.1 \\
$\omega_{3}$ & 1.2 & 1.3 \\
\hline
\end{tabular}

Classes $\omega_{1}, \omega_{2}$, and $\omega_{3}$ correspond, respectively to "black", "dark blue", and "light blue" colors displayed in Fig. 4B. One observes that the shape parameter $p$ fits with the observations: $1.2 \leqslant p \leqslant 2.1$, where $p=2$ corresponds to the classic Gaussian pdf.

Table 4

Kolmogorov test for the GG (whatever $p$ ) and Gaussian noise $(p=2)$ models used for the segmentation on the quadtree of the observed bi-spectral $\left(\mathrm{XS}_{1}-\mathrm{XS}_{2}\right)$ images, displayed on Fig. 4A

\begin{tabular}{lll}
\hline Kolmogorov test $\mathcal{K}_{i}$ on class $\omega_{i}$ & Gaussian modeling & GG modeling \\
\hline $\mathcal{K}_{1}$ & 0.267 & 0.216 \\
$\mathcal{K}_{2}$ & 0.397 & 0.402 \\
$\mathcal{K}_{3}$ & 0.688 & 0.647 \\
\hline
\end{tabular}

These results show that the GG law is a better degradation model to describe the observed radiometry, according to the Kolmogorov criterion.

$$
\widehat{z}(s)=A_{i} \ln \left(y_{s}^{(1)}-\mathrm{XS}_{1}^{\infty}\right)+B_{i} \ln \left(y_{s}^{(2)}-\mathrm{XS}_{2}^{\infty}\right)+C_{i} .
$$

where $A_{i}, B_{i}$ and $C_{i}$ are the bathymetry model parameters to estimate, $\widehat{z}(s)$ denotes the water depth estimated at point $s, \mathrm{XS}_{c}^{\infty}$ holds for the lower radiometry bound, corresponding to high water depth for sensor $c$, and $y_{s}^{(1)}$ and $y_{s}^{(2)}$ stand for radiometry values from channel $\mathrm{XS}_{1}$ and $\mathrm{XS}_{2}$, respectively. For notation convenience, we will write $\widetilde{y}_{c}(s)=\ln \left(y_{s}^{(c)}-\mathrm{XS}_{c}^{\infty}\right)$ with $c \in\{1,2\}$. We adopt a robust estimation technique [26,27] to estimate $A_{i}, B_{i}$, and $C_{i}$ without being affected by the presence of outliers. The estimation is based on $n$ in situ reliable depth measurements $z(l)$, $l=1, \ldots, n$, (as illustrated in Fig. 4B), which leads us to minimize the following function:

$$
\varepsilon\left(A_{i}, B_{i}, C_{i}\right)=\sum_{l=1}^{n} \rho\left(r_{l}\right) \text { with } r_{l}=z(l)-\widehat{z}(l),
$$

where $\widehat{z}(l)$ is the depth value supplied by the model (21) and $\rho$ is a robust estimator. More precisely, we have

$$
\varepsilon\left(A_{i}, B_{i}, C_{i}\right)=\sum_{l=1}^{n} \rho\left(z(l)-A_{i} \widetilde{y}_{1}(l)-B_{i} \widetilde{y}_{2}(l)-C_{i}\right),
$$

where $\rho\left(r_{l}\right)$ is M-estimator able to handle the presence of outliers [26,27]. We have considered the Andrews function defined by:

$$
\rho(r)= \begin{cases}4 \sin ^{2}\left(\frac{r}{2 \alpha}\right) & \text { if }|r|<\pi \alpha \\ 4 & \text { otherwise. }\end{cases}
$$


Its influence function $\Psi(r)=\frac{\mathrm{d}}{\mathrm{d} r} \rho(r)$ is given by

$$
\Psi(r)= \begin{cases}\frac{2}{\alpha} \sin \frac{r}{\alpha} & \text { if }|r|<\pi \alpha \\ 0 & \text { otherwise. }\end{cases}
$$

The minimization of function $\varepsilon$ is achieved by iterated reweighted least-square (IRLS) technique [28]. It amounts to the succession of weighted linear problems:

$$
\left[\begin{array}{ccc}
\sum_{l} v_{l} \widetilde{y}_{1}^{2}(l) & \sum_{l} v_{l} \widetilde{y}_{1}(l) \widetilde{y}_{2}(l) & \sum_{l} v_{l} \widetilde{y}_{1}(l) \\
\sum_{l} v_{l} \widetilde{y}_{1}(l) \widetilde{y}_{2}(l) & \sum_{l} v_{l} \widetilde{y}_{2}^{2}(l) & \sum_{l} v_{l} \widetilde{y}_{2}(l) \\
\sum_{l} v_{l} \widetilde{y}_{1}(l) & \sum_{l} v_{l} \widetilde{y}_{2}(l) & \sum_{l} v_{l}
\end{array}\right]\left[\begin{array}{c}
A_{i} \\
B_{i} \\
C_{i}
\end{array}\right]=\left[\begin{array}{l}
\sum_{l} v_{l} \widetilde{y}_{1}(l) z(l) \\
\sum_{l} v_{l} \widetilde{y}_{2}(l) z(l) \\
\sum_{l} v_{l} z(l)
\end{array}\right],
$$

where weights $v_{l}=\left(\Psi\left(r_{l-1}\right) / r_{l-1}\right)$ are evaluated w.r.t. previous residuals $r_{l-1}$ [28]. The estimation proceeds by alternatively updating the weights and solving (26) as summarized in Table 6.

In Table 5 we report the means and standard deviations (in meter) of water depth values computed for the three classes corresponding to the observation of Fig. 4A and its segmentation displayed in Fig. 4B. Let us notice that these results are in good agreement with the different depth levels evenly spaced $(4.2,10.4$, and $17.5 \mathrm{~m})$ in average.

Table 5

Mean and standard deviation (in meter) of computed water depth for the three classes (Fig. 4B)

\begin{tabular}{lll}
\hline class $\omega_{i}$ & $\mu$ & $\sigma$ \\
\hline$\omega_{1}$ & 17.5 & 4.1 \\
$\omega_{2}$ & 10.4 & 3.0 \\
$\omega_{3}$ & 4.2 & 2.0 \\
\hline
\end{tabular}

Table 6

IRLS procedure for the estimation of the bathymetric model parameters for a given class $\omega_{i}$ of the sea-segmentation

1. Initialization: $v_{l}^{[0]}=1$

2. At the $k$ th iteration:
(a) Solve Eq. (26) with weights $v_{l}^{[k]}$ (parameter values $\left(A_{i}^{[k]}, B_{i}^{[k]}, C_{i}^{[k]}\right)$ ).
(b) Compute $\widehat{z}^{[k]}(l)$ for available in situ measurements.
(c) Update weights $v_{l}^{[k+1]}=\Psi r_{l}^{[k]} / r_{l}^{[k]}$
(d) Stop the procedure when convergence is reached.

Table 7

Errors between true and estimated water depths for the Gaussian and the GG noise models

\begin{tabular}{lll}
\hline Norms (in meter) & $\mathcal{L}_{1}$ & $\mathcal{L}_{2}$ \\
\hline$K=1$ & 1.96 & 2.88 \\
$K=3$ with Gaussian modeling & 1.75 & 2.52 \\
$K=3$ with GG modeling & 1.73 & 2.48 \\
\hline
\end{tabular}

$K=1$ corresponds to a single "sea area" (no segmentation on the quadtree, single inversion model for all the sea area: this is the method used until now by the EPSHOM cartography office). 
Fig. 4C shows the nautical chart generated using the piecewise bathymetric model finally estimated. Linear artifacts are visible but they are already present in the SPOT image and originate from the behavior of the satellite sensors.

The red crosses in Fig. 4B correspond to in situ bathymetry measurements which are used to estimate parameters $A_{i}, B_{i}$, and $C_{i}$ for each class $\omega_{i}, i=1, \ldots, K$. Table 7

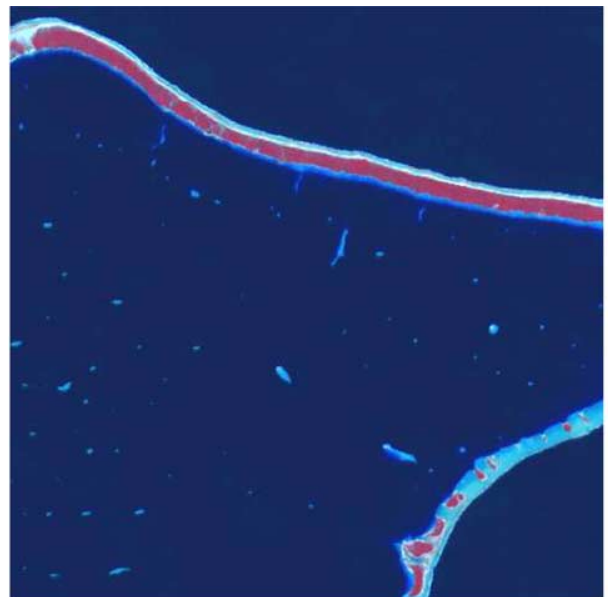

A

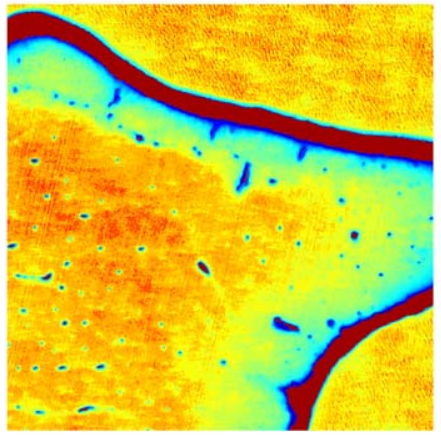

C

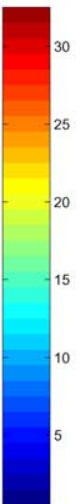

Fig. 5. (A) Observed $512 \times 512$ SPOT Image. (B) Image after segmentation on the quadtree. (C) Water depth estimated (meter). (D) Nautical chart. A lot of corral pinnacles, dangerous for the navigation safety and difficult to locate because of their small sizes, are well detected and classified with our approach. Thus, the unsupervised processing chain we proposed is of great interest to process a large amount of data and allows fast updating of nautical charts.

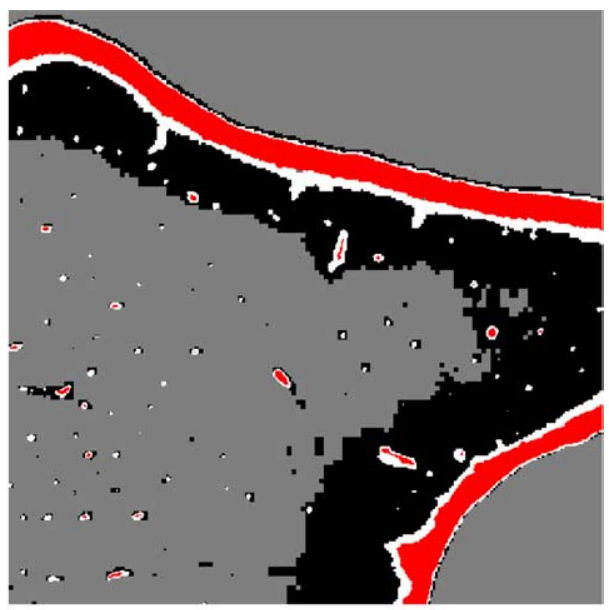

B

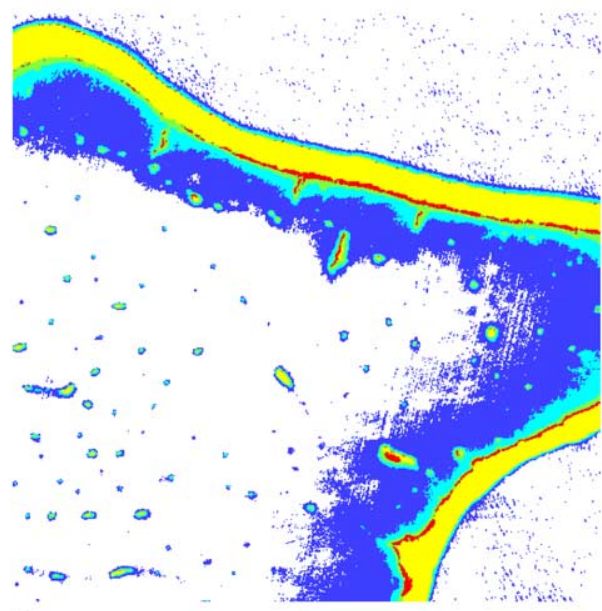

D

ground $\quad 0-5 \mathrm{~m} \quad 5-10 \mathrm{~m} \quad 10-15 \mathrm{~m} \quad 15-20 \mathrm{~m} \quad>20 \mathrm{~m}$ 
reports $\mathcal{L}_{1}$ and $\mathcal{L}_{2}$ error norms between these in situ measurements and estimated water depths at the same locations. The same set of in situ bathymetry measurements (90 points for each class) are considered for the two segmentation maps obtained with the Gaussian model $(p=2)$ and the GG models (whatever $p$ ).

Compared to the classical bathymetric inversion procedure, where one single bathymetric model is learned and used for all the sea area (i.e., $K=1$ ), our approach

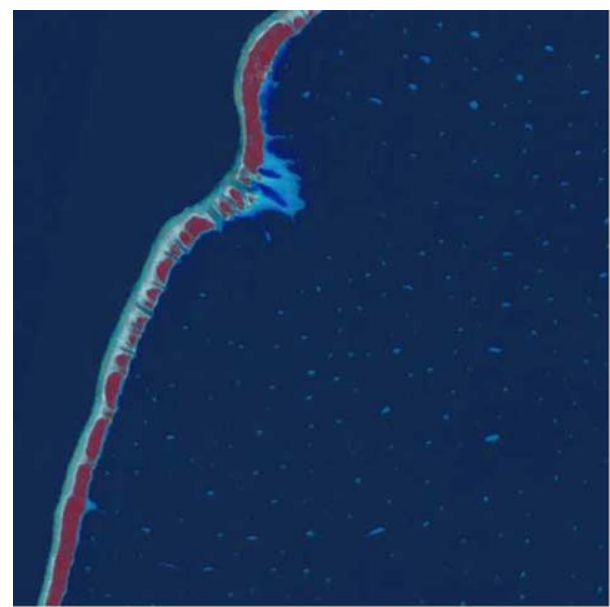

A

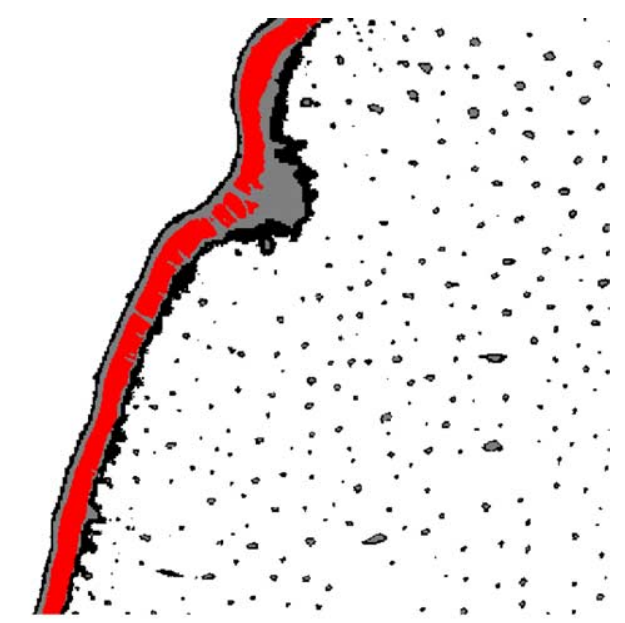

$\mathrm{B}$
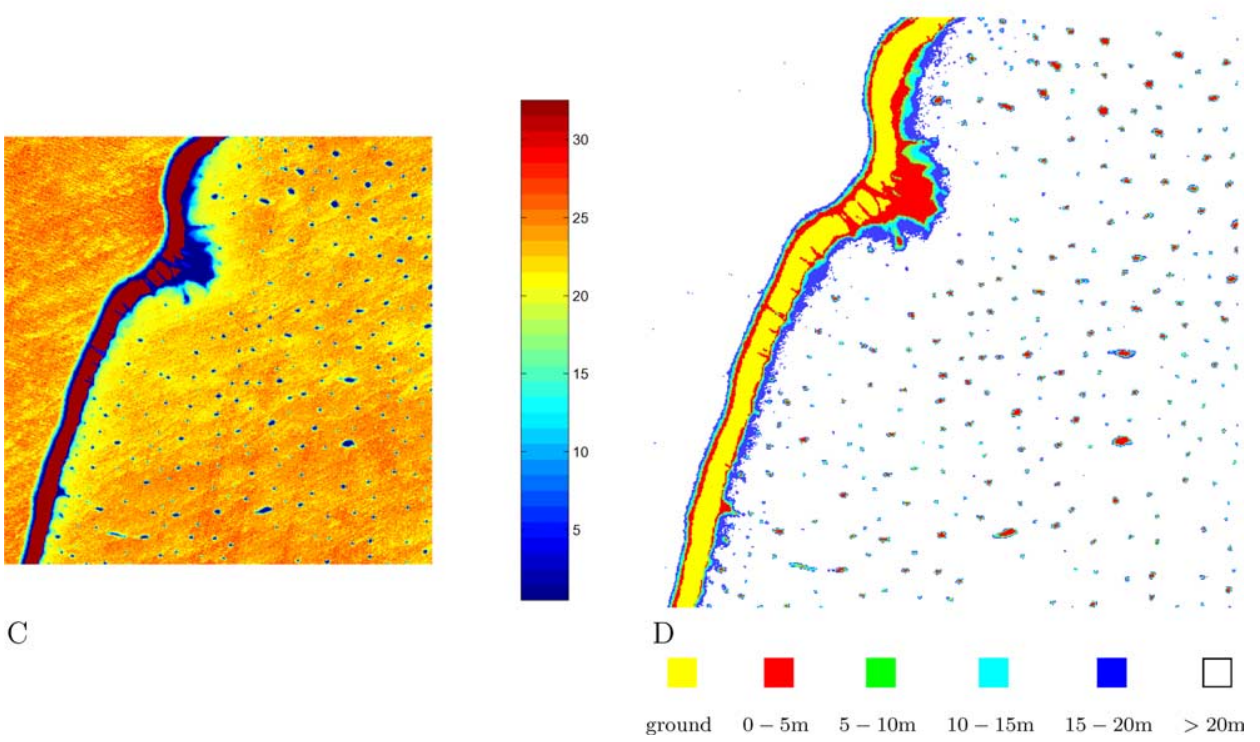

C

ground $\quad 0-5 \mathrm{~m} \quad 5-10 \mathrm{~m} \quad 10-15 \mathrm{~m} \quad 15-20 \mathrm{~m} \quad>20 \mathrm{~m}$

Fig. 6. (A) Observed $512 \times 512$ SPOT Image. (B) Image after segmentation on the quadtree. (C) Water depth estimated (meter). (D) Nautical chart. The processing scheme exhibits rapid convergence properties and is well suited to automatic extraction of features from a large variety of images: the full processing time using a PC-Pentium III bi-processor ( $2 \mathrm{GHz}, 4 \mathrm{Go}$ of $\mathrm{RAM})$ is about $3 \mathrm{~min}$. 
involving an image segmentation into different classes and the estimation of a bathymetry model for each segmentation class, improves the accuracy of the computed water depth map.

\subsubsection{Experimental results}

We tested the classification chain on some real SPOT images (Figs. 4A, 5A and 6A). After extraction of maritime areas by a Markov chain algorithm [3], we have segmented those areas into three classes. This is a good tradeoff between water depth level and available in situ measurements. For other applications, such a segmentation process is obviously faster than other methods for a high number of classes and sensors. Let us quickly remind the different steps of the proposed algorithm for the water depth recover. After a pre-treatment allowing to separate sea areas from the others [3], we adopt a hierarchical Markovian modeling on a quadtree (Fig. 1) to classify the sea zones into different classes corresponding to different water depths (Figs. 5B and 6B). The noise model is based on a generalized Gaussian pdf, allowing to take into account a large variety of distributions and to better fit the model with the observations (as shown in Tables 3, 4 and 7). The outline of the overall unsupervised segmentation algorithm on the quadtree is summarized in Table 1 whereas the parameter initialization and updating is described in Table 2. Then, the last step consists in the reconstruction of the water depth which algorithm is given in Table 6 . This step requires a training set of in situ bathymetric measurements (like in Fig. 4B) and yields to Fig. 5C or Fig. 6C. The nautical chart is then generated by clustering the water depths within a five-meter quantification (see Figs. 4C, 5D and 6D).

The full processing time for an image of size $512 \times 512$ pixels using a PC - Pentium III bi-processor ( $2 \mathrm{GHz}, 4 \mathrm{Go}$ of RAM) is about $3 \mathrm{~min}$.

\section{Conclusion}

We have presented a novel and efficient approach to segment multispectral images with non-Gaussian noise in an unsupervised way. The image noise model exploits Generalized Gaussian distributions and the prior in-scale-Markov model is defined over a quadtree. The GG modeling we have proposed is very attractive to cope with correlated multispectral data. We have demonstrated its efficiency in the context of water depth estimation from SPOT multispectral images: we have validated our method on real data formed by SPOT images and in situ measurements provided by a previous oceanographic campaign and also utilized as ground truth. The segmentation of the sea areas into different depth classes based on multispectral radiometry data allows us to estimate several meaningful sets of parameters defining a global adaptative bathymetric model. It involves the unsupervised classification of the "sea areas" into homogeneous regions. This leads to accurate water depth measurements, demonstrating the feasibility and efficiency of an automatic processing able to handle simultaneously large amounts of data.

The hierarchical Markovian model we have adopted, is a general framework to deal with multispectral and/or multiresolution data. In that sense, this structure is 
well adapted to further extensions integrating observations at different resolutions or from other sensors.

\section{Acknowledgments}

The authors thank the EPSHOM (Etablissement Principal des Services Hydrographiques et Océanographiques de la Marine, Brest) for financial support and fruitful discussions. A web interface using these algorithms is available at http:// picabia.u-strasbg.fr/lsiit/perso/collet/Perso/ColletPerso.htm.

\section{References}

[1] P. Rostaing, J.-N. Provost, C. Collet, Unsupervised multispectral image segmentation using generalized Gaussian noise model, in: Proc. International Workshop EMMCVPR'99 : Energy Minimisation Methods in Computer Vision and Pattern Recognition, vol. LNCS 1654, Springer Verlag, York, UK, 1999, pp. 141-156.

[2] J.M. Laferté, P. Pérez, F. Heitz, "Discrete Markov image modeling and inference on the quad-tree," INRIA Research Report, no. 1198, July (1998).

[3] J.N. Provost, C. Collet, P. Pérez, P. Bouthemy, "Unsupervised multispectral segmentation of spot images applied to nautical cartography," in IEEE-EURASIP Workshop on Nonlinear Signal and Image Processing (NSIP’99), Antalya-Turkey, June (1999).

[4] A. Chardin, P. Pérez, "Semi-iterative inferences with hierarchical energy-based models for image analysis," Proc. International Workshop EMMCVPR'99: Energy Minimisation Methods in Computer Vision and Pattern Recognition, vol. LNCS 1654 (1999).

[5] W. Pieczynski, J. Bouvrais, C. Michel, "Unsupervised bayesian fusion of correlated sensors," in First International Conference on Multisource-Multisensor Information Fusion, Las Vegas, Nevada, USA, June 6-9 (1998).

[6] F. Salzenstein, W. Pieczynski, Parameter estimation in hidden fuzzy markov random fields and image segmentation, Graph. Models Image Process. vol. 59 (4) (1997) 205-220.

[7] J.-P. Delmas, An equivalence of the em and ice algorithm for exponential family, IEEE Trans. Signal Process. vol. 45 (3) (1997) 2613-2615.

[8] N. Giordana, W. Pieczynski, Estimation of generalized multisensor hidden Markov chains and unsupervised image segmentation, IEEE Trans. Pattern Anal. Mach. Intell. vol. 19 (5) (1997) 465-475.

[9] C. Graffigne, F. Heitz, P. Pérez, F. Prêteux, M. Sigelle, J. Zerubia, Hierarchical Markov random field models applied to image analysis: a review, in SPIE Neural Morphological and Stochastic Methods in Image and Signal Processing, San Diego, 10-11 July, vol. 2568 (1995) 2-17.

[10] Z. Kato, M. Berthod, J. Zérubia, A hierarchical Markov random field model and multitemperature annealing for parallel image classification, Graph. Models Image Process. vol. 58 (1) (1996) 18-37.

[11] M. Mignotte, C. Collet, P. Pérez, P. Bouthemy, Bayesian inference and optimization strategies for some detection and classification problems in sonar imagery, in Non linear image processing-SPIE Conference 3646, San Jose, USA, January 1999, vol. 3646-02, pp. 14-27.

[12] C.A. Bouman, M. Shapiro, A multiscale random field model for Bayesian image segmentation, IEEE Trans. Image Process. vol. 3 (2) (1994) 162-177.

[13] J.-M. Laferté, P. Pérez, F. Heitz, Discrete markov image modeling and inference on the quad-tree, IEEE Trans. Image Process. vol. 9 (3) (2000) 390-404.

[14] M. Luettgen, Image Processing with Multiscale Stochastic Models, Phd thesis, MIT Laboratory of Information and Decision Systems, May 1993.

[15] D.R. Lyzenga, Passive remote sensing techniques for mapping water depth and bottom features, J. Appl. Opt. vol. 17 (3) (1978) 379-383. 
[16] D.R. Lyzenga, Remote sensing of bottom reflectance and water attenuation parameters in shallow water using aircraft and Landsat data, in: IEEE Trans. Geosci. Remote Sens., vol. 2, 1983, pp. 77-82.

[17] W. Pieczynski, Champs de Markov cachés et estimation conditionnelle itérative, Traitement du signal vol. 11 (2) (1994) 141-153.

[18] M. Kanefsky, J.B. Thomas, On polarity detection schemes with non-Gaussian inputs, in: J. Franklin Inst., vol. 280, 1965, pp. 120-138.

[19] C.S. Regazzoni, A. Tesei, The asymmetric generalized gaussian funcion: a new hos-based model for generic noise pdfs, IEEE Workshop Signal Process., Corfu, Grecia (1996).

[20] P. Moulin, J. Liu, Analysis of multiresolution image denoising schemes using generalized-gaussian priors, Proc. IEEE TFTS Symposium, Pittsburgh, PA, Oct. 6-9 (1998) 633-636.

[21] C. Lu, H. Liao, Oblivious watermarking using generalized gaussian, 3rd Inter. Conf. Comp. Vis., Pattern Recognition and Image Processing, Atlantic City, USA, Feb. 28-Mar. vol. II (2000) 260-263.

[22] T.T. Pham, R.J.P. deFigueiredo, Maximum likelihood estimation of a class of non-gaussian densities with application to $l_{p}$ deconvolution, IEEE Trans. Acoustics, Speech Signal Process. vol. 37 (1) (1989) $73-82$.

[23] M. Abramowitz, I.A. Stegun, Handbook of Mathematical Functions, Dover, NY, 1970

[24] K. Sharifi, A.L. Garcia, Estimation of shape parameter for generalized gaussian distributions in subband decompositions of video, in: IEEE Trans. Circuits Syst. Video Techn., vol. 5, 1995, pp. 5256.

[25] L. Loubersac, P.Y. Burban, Integrated study of Aitutaki's lagoon (Cook islands) using spot satellite data and in situ measurements: bathymetric modelling, in: Geocarto International, vol. 2, 1991, pp. $31-37$.

[26] J.-M. Odobez, P. Bouthemy, Robust multiresolution estimation of parametric motion models, J. Vis. Commun. Image Represent. vol. 6 (4) (1995) 348-365.

[27] P. Meer, D. Mintz, A. Rosenfeld, D.Y. Kim, Robust regression methods for computer vision: a review, Int. J. Comput. Vis. vol. 6 (1) (1990) 59-70.

[28] J. Huber, Robust Statistics, Wisley, New York, 1981.

[29] S. Banks, Signal Processing, Image Processing and Pattern Recognition, Prentice Hall, Englewood Cliffs, NJ, 1990. 